\title{
Anterior Cruciate Ligament Reconstruction: The Long Road from Science to Clinical Relevance
}

\author{
Matthieu Ollivier, $\mathrm{MD}, \mathrm{PhD}$ \\ Department of Orthopaedic Surgery, Institute for Movement and Locomotion, Sainte Marguerite Hospital, Marseille, France
}

The anterior cruciate ligament (ACL) has entertained scientific and clinical minds since Robert Adams described the first clinical case of ACL rupture in 1837. Over the next 100 years, surgeons have, thus, experimented different techniques, graft sources and postoperative protocols ${ }^{1)}$. But only arthroscopic strategies, initially developed by Kenji Takagi and Masaki Watanabe and popularized by Jackson and Dandy, firmly established ACL reconstruction as a common procedure within the realm of most surgeons' skills ${ }^{1)}$. From this starting point, techniques and procedures moved back and forth over a decade of fluctuation: after a strong mechanical cycle, the principle of anatomic ACL reconstruction, aiming at the functional restoration of native ACL dimensions and insertion sites, has been re-introduced, suspending the concept of isometric graft placement ${ }^{2}$. Then, after 10 years of popularity, double-bundle reconstruction lost its popularity in favor of "more biomechanical procedures" such as combined extra- and intra-articular techniques. Hall and Platell ${ }^{3)}$ reported in their Lancet paper, that the half-life of truth in surgical procedures was 45 years, but it might be even shorter in sports medicine and ACL reconstruction. The main issue for clinicians today is to criticize scientific facts through a paucity of level I clinical studies (using limited tools to compare multifactorial surgical successes) and an abundant amount of mechanical studies (using

Correspondence to: Matthieu Ollivier, $\mathrm{MD}, \mathrm{PhD}$

Department of Orthopaedic Surgery, Institute for Movement and Locomotion, Sainte Marguerite Hospital, 20 Avenue Viton, Marseille 13009, France

Tel: +33-0491745011, Fax: +33-0491745001

E-mail: ollivier.mt@gmail.com

This is an Open Access article distributed under the terms of the Creative Commons Attribution Non-Commercial License (http://creativecommons.org/licenses/by-nc/4.0/) which permits unrestricted non-commercial use, distribution, and reproduction in any medium, provided the original work is properly cited. robust and validated testing platforms to estimate millimeters, degrees or newton-based differences between anatomical specimens). As mentioned by $\mathrm{Smith}^{4)}$ in his recent editorial comment, we, orthopedic surgeons, might actually be blinded by complex biomechanical studies when more and more evidence is asked to support our simple clinical feelings. The "road" is long and future surgeons will judge our walk.

The present issue of Knee Surgery \& Related Research focuses on the ACL, with a special interest in recent diagnostic tools, biomechanical aspect of the reconstruction and postoperative clinical outcomes in patients older than 40 years.

Ryu et al. aimed to compare, in their in vivo study including 40 patients with acute ACL injuries, the accuracy of the GNRB arthrometer (Genourob), Lachman test, and Telos device $(\mathrm{GmbH})$ to diagnose ACL ruptures. They concluded that all diagnostic tools were informative but depicted the higher diagnostic capability of the GNRB arthrometer to assess side-to-side laxity differences. It is important to note that the GNRB is probably the most accurate clinical diagnostic tool; however, definitive validation still needs to be done as most of the studies involving healthy and uninjured knees disagree its diagnostic capability in a normal $\mathrm{knee}^{5,6)}$.

Two ex vivo studies are also published in this issue. Kim et al. investigated anatomical morphometric parameters of ACL femoral insertion site. Eighteen embalmed cadaveric knees (mean age, 70 years) were examined to determine the shape and position of the ACL femoral footprint using C-arm analysis. The authors confirmed Smigielski et al's anatomical dissection, breaking the concept of double-bundle, and thus, demonstrating the ACL's ribbon shape ${ }^{7,8)}$. In their description, the ACL was a flat single bundle with an average length of $34 \mathrm{~mm}$ and an average width of $9 \mathrm{~mm}$. Its center was positioned slightly more anterior and distal than previously described positions, which might be useful for 
determining optimal anatomical landmarks in the Asian population.

Kim et al. aimed to evaluate isometry of in vivo anatomic ACL reconstruction. They found that anatomic reconstruction of the ACL conduces to relatively anisometric graft: the greater the flexion angle of the knee, the shorter the graft length in the joint. It is important to note that those finding are congruent with most studies focusing on in vivo native ACL behavior during knee flexion. The native ACL is deeply anisometric, tense in extension and loose in flexion ${ }^{9)}$. Those results also confirmed that aiming for an isometric ACL graft will result in non-physiologic knee kinematics.

Because the average age and life expectancy are rising, the physical activity level in the elderly population is increasing and ACL injuries are becoming more frequent in middle-aged patients. Traditionally, these patients have been treated conservatively since the benefits of the surgical procedure would not be balanced by the risks. However, recent literature ${ }^{10)}$ demonstrated that conservative treatment results in higher rates of residual instability and adverse clinical outcomes. In a meta-analysis, Kim et al. included seven studies specifically evaluating comparative series of patients over 40 years and under 40 years. They confirmed no significant differences in terms of validated ACL scoring systems or mechanical outcomes between the two groups of patients being older or younger than 40 years.

\section{References}

1. Schindler OS. Surgery for anterior cruciate ligament deficiency: a historical perspective. Knee Surg Sports Traumatol Arthrosc. 2012;20:5-47.

2. Fu FH, Schulte KR. Anterior cruciate ligament surgery 1996: state of the art? Clin Orthop Relat Res. 1996;(325):19-24.

3. Hall JC, Platell C. Half-life of truth in surgical literature. Lancet. 1997;350:1752.

4. Smith PA. Editorial commentary: filling the void in translational research for lateral extra-articular tenodesis for anterior cruciate ligament reconstruction: are we saturated with biomechanical studies? Arthroscopy. 2018;34:261-3.

5. Mouarbes D, Cavaignac E, Chiron P, Berard E, Murgier J. Evaluation of reproducibility of robotic knee testing device (GNRB) on 60 healthy knees. J Orthop. 2018;15:94-8.

6. Murgier J, Beranger JS, Boisrenoult P, Steltzlen C, Pujol N. Prospective comparative study of knee laxity with four different methods in anterior cruciate ligament tears. Int Orthop. 2018 Feb 2 [Epub]. http://dx.doi.org/10.1007/s00264018-3791-4.

7. Noailles T, Boisrenoult P, Sanchez M, Beaufils P, Pujol N. Torsional appearance of the anterior cruciate ligament explaining "ribbon" and double-bundle concepts: a cadaverbased study. Arthroscopy. 2017;33:1703-9.

8. Smigielski R, Zdanowicz U, Drwiega M, Ciszek B, Ciszkowska-Lyson B, Siebold R. Ribbon like appearance of the midsubstance fibres of the anterior cruciate ligament close to its femoral insertion site: a cadaveric study including 111 knees. Knee Surg Sports Traumatol Arthrosc. 2015;23:3143-50.

9. Guenoun D, Vaccaro J, Le Corroller T, Barral PA, Lagier A, Pauly V, Coquart B, Coste J, Champsaur P. A dynamic study of the anterior cruciate ligament of the knee using an open MRI. Surg Radiol Anat. 2017;39:307-14.

10. Iorio R, Iannotti F, Ponzo A, Proietti L, Redler A, Conteduca F, Ferretti A. Anterior cruciate ligament reconstruction in patients older than fifty years: a comparison with a younger age group. Int Orthop. 2018;42:1043-9. 